\title{
Correction to: Distributed Autonomous Robotic Systems
}

Roderich Groß, Andreas Kolling, Spring Berman, Emilio Frazzoli, Alcherio Martinoli, Fumitoshi Matsuno and Melvin Gauci

\section{Correction to:}

R. Groß et al. (eds.), Distributed Autonomous Robotic Systems, Springer Proceedings in Advanced Robotics 6, https://doi.org/10.1007/978-3-319-73008-0

The original version of the book was inadvertently published with an incorrect spelling of the editors' affiliations and provided corrected version of preface.

The corrected book has been updated with the changes. 